\title{
Study of Platelet Parameters- Mean Platelet Volume (MPV) and Platelet Distribution Width (PDW) in Type 2 Diabetes Mellitus
}

\author{
Advait Anirudha Joshi ${ }^{1}$ and Janice Jaison ${ }^{2 *}$ \\ 'Undergraduate Student, MIMER Medical College, Talegaon Dabhade, Pune, India \\ ${ }^{2}$ Assistant Professor, Department of Pathology, MIMER Medical College, Talegaon Dabhade, Pune, India
}

\begin{abstract}
Background: Diabetes Mellitus is a global public health problem with morbidity due to complications caused by macrovascular and microvascular disease. Platelet parameters like Mean Platelet Volume (MPV) and Platelet Distribution Width (PDW) are related to platelet size, enzymatic activity and prothrombotic potential. These parameters can be obtained from routine hemogram reports of automated hematology analyzers at a relatively low cost. Fasting blood glucose, postprandial blood glucose, and hemoglobin A1C (HbA1c) are widely used to monitor glycaemic control in patients with DM. The aim of this study is to study Platelet parameters- Mean Platelet Volume (MPV) and Platelet Distribution Width (PDW) in type 2 diabetes and its predictive role in diabetic angiopathies.
\end{abstract}

Methods: The present study was a Case control study carried for a period of 2 months. Statistical analysis was done with appropriate tests.

Result: MPV values were lower in cases than controls. In controls, MPV shows a negative correlation with fasting blood sugar while in cases it shows a positive correlation with fasting blood sugar. MPV showed no association with HbA1c. PDW was high in cases than controls. In controls, negative correlation between PDW and FBS was observed, while in cases, a positive correlation was observed. A strong association between PDW and HbAlc was noted. At a cut off of $9.5 \mathrm{fL}$, MPV had a sensitivity of $78.33 \%$, specificity of $70 \%$ and a positive predictive value of $72.30 \%$. At a cut off of $15 \mathrm{fL}$, PDW showed a sensitivity of $81.66 \%$, specificity of $91.66 \%$ and a positive predictive value of $90.74 \%$.

Conclusion: The present study shows strong association between PDW and HbAlc and so it can be used as an indicator of impending vascular events. Of all the platelet parameters obtained by haematology cell counters, Platelet count is only widely used as investigative tool. Platelet indices can be used as simple and cost effective bio markers for predicting diabetic vascular complications.

\section{Keywords: Diabetes Mellitus, Platelet indices, MPV, PDW}

\section{Introduction}

Diabetes Mellitus is a global public health problem increasing at an alarming rate. By 2030 , about 80 to 87 million people of India will be diabetic and 438 million people $(7.8 \%)$ of the adult population is expected to have diabetes $^{[1]}$ The morbidity associated with long standing diabetes of either type results from complications caused by macrovascular disease and microvascular disease. Macrovascular disease causes accelerated atherosclerosis resulting in increased risk of myocardial infarction, stroke and lower extremity gangrene. Microvascular disease contributes to diabetic retinopathy, nephropathy and neuropathy. ${ }^{[2,3]}$

Many recent studies have reported that haematological indices could be used as indicators contributing to the vascular injury in diabetic patients. ${ }^{[4]}$ Platelets play a vital role in the integrity of normal hemostasis. Large platelet contain dense granules, are metabolically and enzymatically more active than smaller ones and have higher thrombotic potential. Platelet parameters like Mean
Platelet Volume (MPV) and Platelet Distribution Width (PDW) are related to platelet size, enzymatic activity and prothrombotic potential. ${ }^{[5,6,7]}$ These parameters can be obtained by routine hemograms done using automated haematology analyzers at a relatively low cost.

In this study, we aim to compare and analyze the platelet parameters, MPV and PDW in cases and controls with short term and long term markers of glycemic control, namely fasting blood sugar levels and HbA1c levels respectively. Various studies have been done to find the relationship between Type 2 diabetes mellitus and Platelet parametersMPV and PDW in urban population, but studies in the rural population is very less.

The newer hematological analyzers give a variety of platelet parameters which helps in easy detection of change in platelet structure, which may help in early detection of prothrombotic state of the platelets. These can help in diagnosing initiation or progression of diabetic complications. 
The objectives of this study are:

1. To determine platelet parameters - mean platelet volume (MPV) and platelet distribution width (PDW) in subjects with Type $2 \mathrm{DM}$ and compare them with age and sex matched controls without Diabetes.

2. Correlation of the platelet parameters with fasting blood glucose levels and glycosylated hemoglobin (HbA1c) levels.

\section{Materials and Methods}

This was a case control study conducted at a 700 bedded rural general hospital in India. The period of the study was from April 2018 to June 2018. A total of 120 patients were recruited as a part of the study from which 60 were cases and 60 were controls. All patients coming to the outpatient as well as in patient departments of our hospital with Type 2 DM satisfying the inclusion and exclusion criteria were included in the present study. The study was approved by the Institutional Ethical committee of our college. The inclusion Criteria for the cases was patients, 25 years and above, with fasting blood sugar equal to and above $126 \mathrm{mg} / \mathrm{dL}$ while patients, 25 years and above, who had fasting blood sugar equal to or less than $100 \mathrm{mg} / \mathrm{dL}$ were taken as controls. The exclusion criteria included pregnant women, smokers, alcoholics, patients on treatment of antihypertensive drugs, statins, insulin therapy, antiplatelet drugs and anticoagulant therapy. Patients having diseases or conditions which affect platelet size and distribution such as idiopathic thrombocytopenic purpura, acute post streptococcal glomerulonephritis, renal failure, myocardial infarction, ischemic heart disease, cerebrovascular accident, malignancies and hypertension were also excluded from this study.

$2 \mathrm{ml}$ blood was collected in fasting and postprandial conditions in fluoride vacutainer for estimation of blood glucose level and $2 \mathrm{ml}$ blood in EDTA vacutainer for platelet parameters and $\mathrm{HbA} 1 \mathrm{c}$ estimation. HbA1c test was only performed in cases. Fasting and blood glucose levels were estimated by using Spectrophotometric method and platelet parameters (MPV and PDW) were obtained using 3 part Alere h 380 and 5 part XS-800i automated blood cell counter. HbA1c levels were estimated by NycoCard Boronate affinity assay method.The normal laboratory values were considered as follows:

1. MPV- 7.4-10.4 fL

2. PDW- 9-13 fL

3. HBA1c- According to ADA( American Diabetic Association), the criteria for the diagnosis of diabetes is $\mathrm{HbA} 1 \mathrm{c} \geq$ to $6.5 \%$
In the present study, the blood samples were run on Alere h 380 and XS 800 I haematology analyzers, in which the readings of PDW were obtained in Femtolitres (fl). PDW can be expressed as $\%$ or fl.

The results were entered into Excel (Microsoft), Googlesheets and Statistical Package for the Social Sciences (SPSS), and required biostatistics tests were applied.

\section{Result}

The study consisted of a total of 120 patients, of which 60 were diabetic patients who were the cases, and 60 were non-diabetic individuals, who formed the control group. The male to female ratio of diabetic group was $1: 1$, and that of the control group was $0.8: 1$. The mean age of the diabetic group was $44.42 \pm 1.83$ with a minimum age of 25 years and a maximum age of 83 years; whereas in the control group, the mean age was $54.72 \pm 1.78$. (Table 1)

The mean MPV in the cases was $7.27( \pm 2.42) \mathrm{fL}$, while in the controls it was $10.03( \pm 1.16) \mathrm{fL}$ which was statistically significant. The mean PDW in the cases was $16.66( \pm 2.98)$ fL while in the controls it was $11.78( \pm 2.44) \mathrm{fL}$, which was also statistically significant. $\mathrm{HbA} 1 \mathrm{C}$ was performed only in the cases.

The MPV was lower in cases than in controls, while the PDW was higher in cases than in controls. (Table 2).Due to these inverse trends in controls and cases, distribution of cases and controls was seen separately with respect to fasting blood sugar.

It was observed that MPV decreases with increase in fasting blood sugar in controls. As against this, MPV increases with increase in fasting blood sugar in cases. (Fig.1). Similarly, distribution of PDW with respect to fasting blood sugar was plotted separately for cases and controls (Fig.2). In controls, there is a decrease in PDW with increase in the fasting blood sugar. In cases, there is an increase in PDW with increase in the fasting blood sugar.

Further analysis was done to confirm these trends. We carried out a Spearman's rank-order correlation between fasting blood sugar and MPV, PDW and HbA1C for the controls and cases. (Table 3 )

In controls, MPV shows a negative correlation with fasting blood sugar, which is statistically significant. (Rho= $\mathbf{- 0 . 2 8 1}, p=\mathbf{0 . 0 3 0}, \mathbf{N}=\mathbf{6 0}$ ) and PDW also shows a negative correlation with fasting blood sugar, which is statistically significant. ( $\mathrm{Rho}=\mathbf{- \mathbf { 0 . 2 8 7 }}, p=\mathbf{0 . 0 2 6}, \mathbf{N}=\mathbf{6 0}$ ). In cases, MPV shows a positive correlation with fasting blood sugar, which is marginally significant. ( $\mathrm{Rho}=\mathbf{0 . 2 4 8}, p=\mathbf{0 . 0 5 6}$, $\mathbf{N}=\mathbf{6 0}$ ), while PDW also shows a positive correlation with fasting blood sugar. However, it is not statistically significant. $(\mathrm{Rho}=0.021, p=0.874, \mathbf{N}=\mathbf{6 0})$ 
HbA1C showed a positive correlation with fasting blood sugar, which was statistically significant. $(\mathrm{Rho}=\mathbf{0 . 6 5 4}, \mathrm{p}=$ $<\mathbf{0 . 0 0 0 5}, \mathrm{N}=60$ )

Using Odds ratio, we calculated the association of $\mathrm{HbA1C}$, with MPV and PDW. The Odds ratio for MPV was 1.2424 with $95 \%$ CI ranging between 0.21 to 7.03 . The significance level was $\mathrm{P}=0.8061$. For $\mathrm{PDW}$ the Odds ratio calculated was 84.00 , with $95 \%$ CI ranging between 8.16 to 863.70 . The significance level was $\mathrm{P}=0.0002$. This indicates strong association between PDW and HbA1C levels.
We calculated the sensitivity and specificity and positive predictive value for MPV and PDW to be used as a diagnostic test. (Table 5). MPV below 9.5 fL were considered as true patients and PDW equal to and above $15 \mathrm{fL}$ were considered as true patients. The above results indicate that PDW is a better marker to predict deterioration of glucoregulation.

\section{Discussion}

Diabetes, particularly DMT2 patients are exposed to increased platelet reactivity due to multifactorial

Table 1: Distribution of Age and Gender across controls and cases.

\begin{tabular}{|c|c|c|c|c|c|}
\hline \multicolumn{3}{|c|}{ Controls } & \multicolumn{3}{|c|}{ Cases } \\
\hline \multirow[t]{2}{*}{ Age } & \multicolumn{2}{|c|}{ Sex } & \multirow[t]{2}{*}{ Age } & \multicolumn{2}{|c|}{ Sex } \\
\hline & Male & Female & & Male & Female \\
\hline $25-30$ & 6 & 6 & $25-30$ & 4 & 1 \\
\hline $31-40$ & 8 & 8 & $31-40$ & 5 & 1 \\
\hline $41-50$ & 3 & 8 & $41-50$ & 3 & 7 \\
\hline $51-60$ & 3 & 6 & $51-60$ & 7 & 9 \\
\hline $61-70$ & 7 & 4 & $61-70$ & 7 & 10 \\
\hline \multirow[t]{2}{*}{$>70$} & 1 & 0 & $>70$ & 4 & 2 \\
\hline & 28 & 32 & & 30 & 30 \\
\hline
\end{tabular}

Table 2: Means of parameters in cases and controls (values expressed in brackets are the values of standard deviation of the means). All p values are significant at $<0.05$.

\begin{tabular}{|c|c|c|c|}
\hline Parameters & $\begin{array}{c}\text { Controls } \\
\text { Mean }( \pm \text { SD) }\end{array}$ & $\begin{array}{c}\text { Cases } \\
\text { Mean }( \pm \text { SD) }\end{array}$ & p values \\
\hline Blood Sugar fasting (mg/dL) & $\begin{array}{c}93.67 \\
( \pm 4.08)\end{array}$ & $\begin{array}{c}188.68 \\
( \pm 63.53)\end{array}$ & -0.00005 \\
\hline HbA1C $(\%)$ & -- & $\begin{array}{c}9.07 \\
( \pm 2.22)\end{array}$ & $<0.00005$ \\
\hline Mean platelet volume - MPV (fL) & $\begin{array}{c}7.27 \\
( \pm 2.42)\end{array}$ & $\begin{array}{c}16.66 \\
( \pm 2.98)\end{array}$ \\
\hline $\begin{array}{c}( \pm 1.16) \\
\text { Platelet distribution width - PDW } \\
(\mathrm{fL})\end{array}$ & $\begin{array}{c}11.78 \\
( \pm 2.44)\end{array}$ & $<0.00005$ \\
\hline
\end{tabular}

Table 3: Spearman's rank-order correlations of fasting blood sugar with mean platelet volume (MPV), platelet distribution width (PDW) and HbA1C. The significant correlations are highlighted.

\begin{tabular}{|c|c|c|c|c|}
\hline & \multicolumn{3}{|c|}{ Fasting blood sugar } \\
\hline & \multicolumn{2}{|c|}{ Controls $(\mathbf{N}=\mathbf{6 0})$} & Rho & Cases (N = 60) \\
\hline & Rho & p value value & 0.056 \\
\hline MPV & -0.281 & 0.030 & 0.248 & 0.874 \\
\hline HDW & -0.287 & 0.026 & 0.021 & $<0.0005$ \\
\hline
\end{tabular}

Table 4: Odds ratio between HbA1c and MPV and between HbA1c and PDW

\begin{tabular}{|c|c|c|}
\hline \multirow{2}{*}{} & \multicolumn{2}{|c|}{ HbA1c } \\
\cline { 2 - 3 } & Odds Ratio & $95 \% \mathbf{C l}$ \\
\hline MPV & 1.24 & 0.21 to7.03 \\
\hline PDW & 84.00 & 8.16 to863.70 \\
\hline
\end{tabular}

Table 5: Results of sensitivity, specificity and positive predictive value for MPV and PDW Values.

\begin{tabular}{|c|c|c|c|}
\hline Parameters & Sensitivity & Specificity & Positive Predictive Value \\
\hline MPV(fL) (cutoff:9.5) & $78.33 \%$ & $70 \%$ & $72.30 \%$ \\
\hline PDW(fL) (cutoff:15) & $81.66 \%$ & $91.66 \%$ & $90.74 \%$ \\
\hline
\end{tabular}



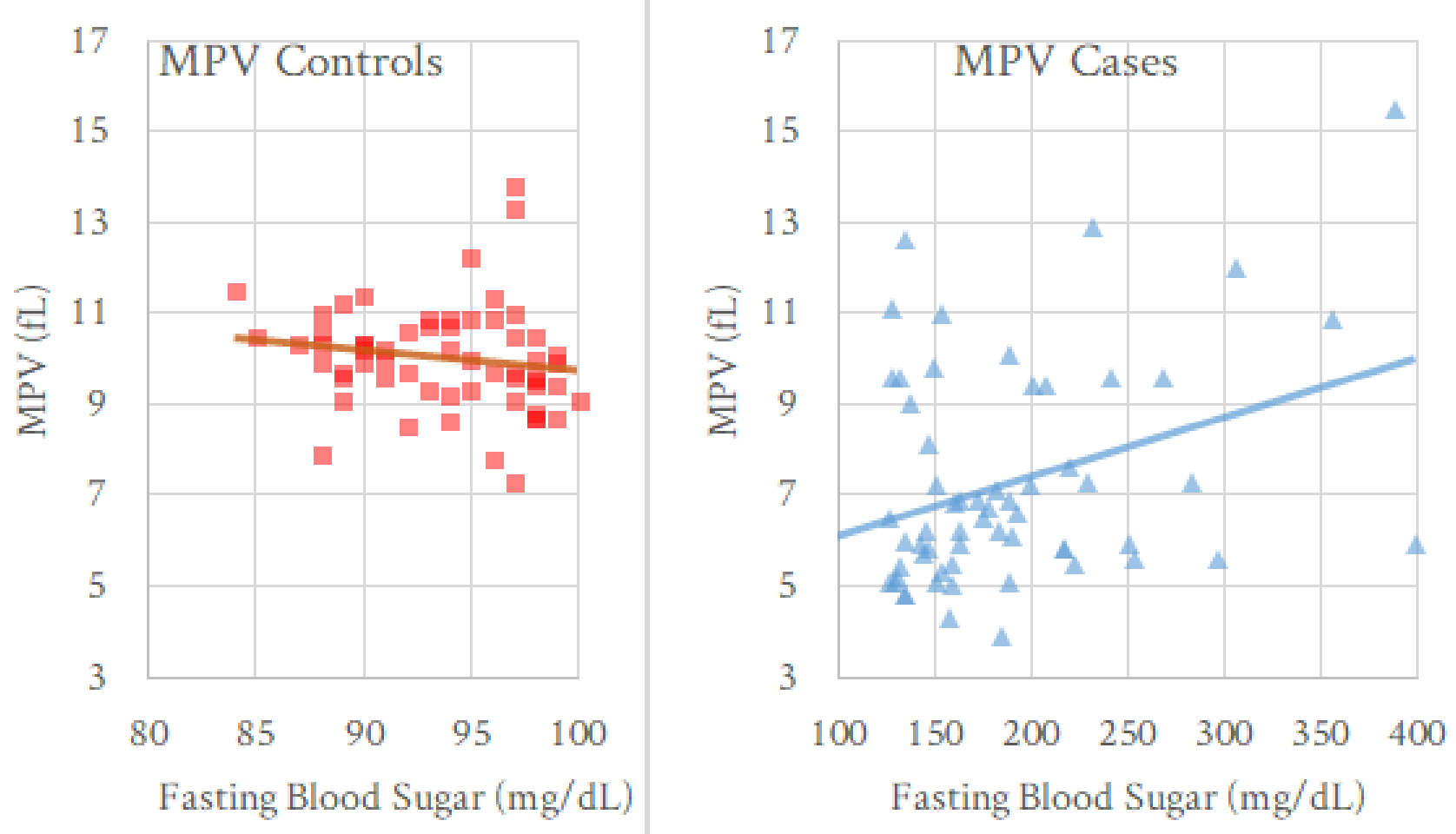

Fig. 1: Scatter plot showing the fasting blood sugar (mg/dL) and mean platelet volume - MPV (fL).
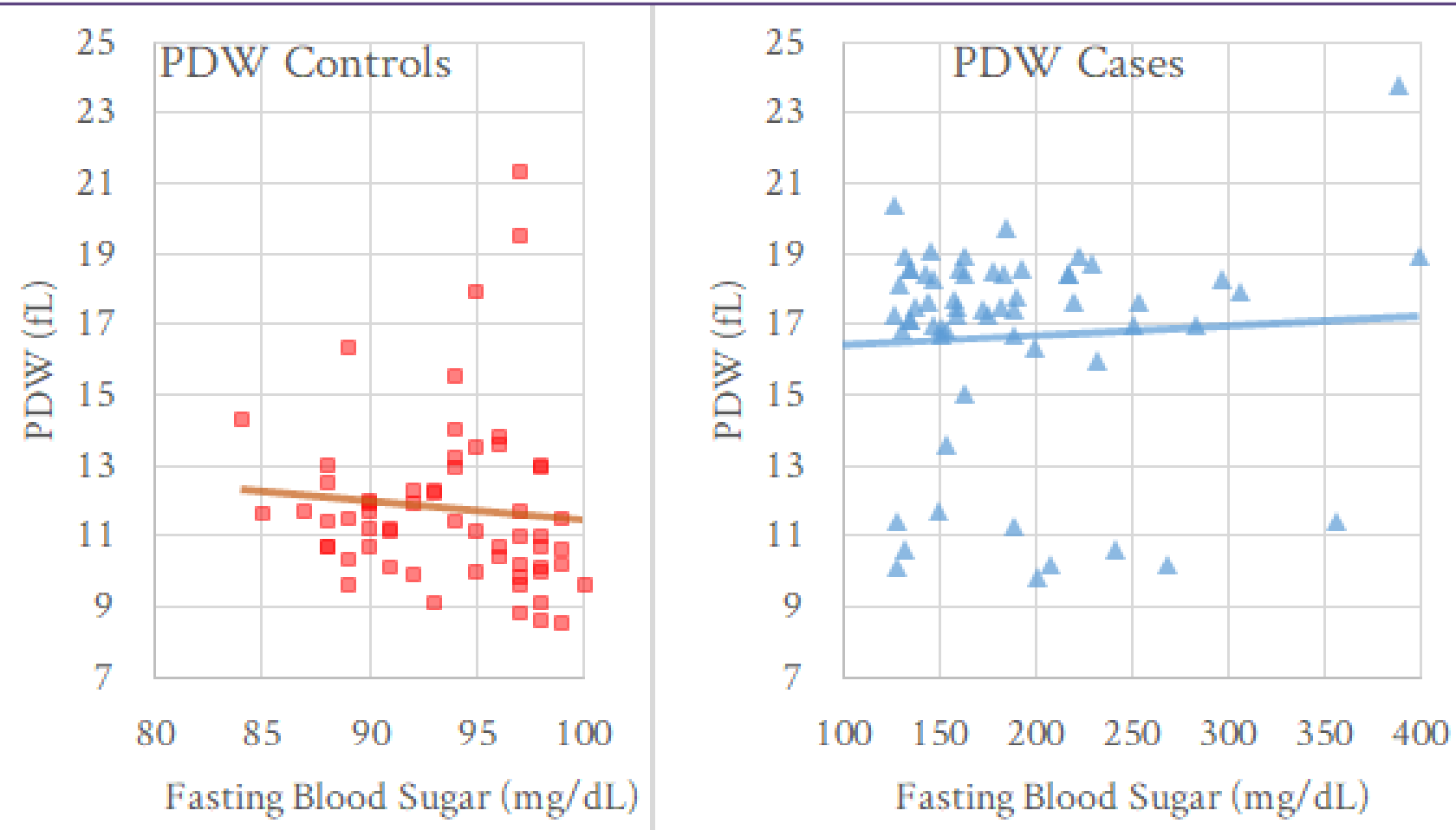

Fig. 2: Scatter plot showing the relation between fasting blood sugar (mg/dL) and platelet distribution width - PDW (fL). 
causes such as metabolic(e.g. hyperglycemia, hypertriglyceridemia), systemic abnormalities (e.g. oxidative stress, inflammation) and insulin resistance. ${ }^{[8]}$ There is increased thrombopoiesis and circulation of large, young and activated platelets which undergo episodes of release of granules, accelerated sequestration and reduced survival. The alterations in platelets in diabetes are increase in its size and hyperactivity.

Mean Platelet Volume (MPV) and Platelet Distribution Width (PDW) are morphometric indices reflecting the size distribution of the peripheral platelet population.

MPV is a parameter used to assess platelet size an it is a potential biomarker of platelet reactivity. The causes of increase in MPV in uncontrolled diabetics could be based on few of the following hypothetical theories:

- Major source of energy for platelets is glucose. In DM, due to chronic hyperglycemia, platelets are overwhelmed with glucose and subjected to synthesis of glycogen contributing to increase in MPV.

- Osmotic swelling of the platelets leads to increase in MPV

- MPV also depends upon the platelet granule content, number of glycoprotein molecules on platelet membrane and thromboxane synthesizing capacity. ${ }^{[9]}$

Larger platelets are younger, hyperactive and more aggregable when compared with smaller platelets because they secrete more serotonin and beta thromboglobulin, contain denser granules and produce more thromboxane A2 producing a procoagulant effect leading to thrombotic vascular complications. This suggests the association between the platelet size and diabetic vascular complications, thus indicating alterations in MPV reflect the state of thrombogenesis. ${ }^{[10]}$

PDW can directly measure the variability of platelet size. Increased endothelial damage is seen in diabetes mellitus, which reduces the survival of platelets and increases turnover of younger platelets. PDW is affected by the increased number and size of pseudopodia. Activated platelets differ in size due to change from a discoid to a sphearical and pseudopodia formation which results in a change in PDW. ${ }^{[9]}$

A significant increase in the mean MPV is seen in cases than in controls in most of the available literature..$^{[3,4,5,7,8,9,10,11,12,13]}$ The relation between MPV and Type 2 Diabetes Mellitus was first reported by Sharpe and Trinick who found a significant increase of MPV in diabetic compared with non diabetic patients. ${ }^{[8]}$ In a study done by Akinbami A et al, MPV was lower in diabetics than in non diabetic controls. However, this difference was not statistically significant. $(p=0.593)$. This was stated to be due to the fact that majority of diabetics in the study group were on treatment, particularly with antiplatelet medications like Clopidogrel and vasopressin for varying durations. In the present study, the mean MPV in the cases was $7.27( \pm 2.42) \mathrm{fL}$, while in the controls it was $10.03( \pm 1.16) \mathrm{fL}$ which was statistically significant, indicating that patients with diabetes had a lower MPV as compared to the control group. These findings are comparable with the studies done by Akinbami A et al. ${ }^{[15]}$

There are reports that both anticoagulants and platelet aggregation inhibitors directly or indirectly influence the platelet indices. Decreased MPV is noted in tuberculosis during disease exacerbation, ulcerative colitis, SLE in adults and in different neoplastic diseases. ${ }^{[23]}$ Decreased MPV is also associated with Iron deficiency Anaemia. ${ }^{[24]}$ In a study carried out by S Periasamy et al in a rural population, the incidence of anaemia in Type 2 Diabetes mellitus patients was $74 \%$ of which $35 \%$ of the patients had Microcytic Hypochromic Anaemia. ${ }^{[2]}$ Preanalytical factors such as storage time and temperature, type of anticoagulant used and methodology of measurement may also influence MPV value. ${ }^{[22,25]}$

In the present study,patients with diabetes had a lower MPV as compared to the control group. Our findings are contradictory to the findings of most of earlier studies. Our study was based in a rural population, where anaemia is common. Factors like prevalence of iron deficiency anaemia, medication, duration of diabetes, may influence the results of MPV. However, the reasons for the decrease in MPV was beyond the scope of this study.

In controls, MPV shows a negative correlation with fasting blood sugar, which is statistically significant. ( $\mathrm{Rho}=-0.281$, $p=0.030, \mathrm{~N}=60)$. This finding is comparable with the findings of Jabeen F et al and Agrawal et al. ${ }^{[3,16]}$ The study done by Binita Shah et al, further explains that significant correlation of MPV with the degree of glycemic control was observed only in the diabetic population and not in the nondiabetic population. ${ }^{[13]}$ In cases, MPV shows a positive correlation with fasting blood sugar, which is marginally significant. ( $\mathrm{Rho}=0.248, p=0.056, \mathrm{~N}=60$ ). This finding is comparable with the studies done by Damira et al, Kamilla et al and Binita Shah et al. ${ }^{[12,13,17]}$

Studies have used HBA1c as a discriminator to divide diabetic patients into 2 groups to compare the platelet indices. The cutoff of $\mathrm{HbA} 1 \mathrm{c}$ varied in the different studies. Some have used $6.5 \%$ as a cut off, while few studies have used $7 \%$ as cut off. A significant positive correlation between MPV and HbAlc was noted by Lavanya et al, Jaman S et al, Anandhalakshmi et al, Kamilla et al, Sharma 
$M$ et al. ${ }^{[8,9,10,12,13]}$ Using Odds ratio, we calculated the association of $\mathrm{HbA} 1 \mathrm{c}$ with MPV. The Odds ratio for MPV was 1.2424 with $95 \%$ CI ranging between 0.21 to 7.03 . The significance level was $\mathrm{P}=0.8061$. Hence in our study we could not prove association between $\mathrm{HbA} 1 \mathrm{c}$ and MPV.

Studies by Lavanya Rajagopal et al indicated that the mean PDW among uncontrolled diabetics was much higher than those with controlled diabetes and non-diabetics. ${ }^{[9]}$ Ansari et al, Bhanukumar et al, Sharma et al, Biadgo B et al, Jabeen F et al, Kamilla et al also observed higher values of PDW in diabetic patients when compared to non-diabetics . These differences were found to be statistically significant. ${ }^{[3,4,5,10,11,13]}$ Buch A et al observed statistically significant difference in PDW between diabetics with complications, diabetics without complications and nondiabetic groups $(p<0.0001)$. PDW was statistically increased in diabetic retinopathy and nephropathy. ${ }^{[7]}$ According to Anandhalakshmi et al, PDW was higher in diabetics but was not statistically significant when compared with the control group. ${ }^{[12]}$ In the present study, the mean PDW in the cases was $16.66( \pm 2.98) \mathrm{fL}$ while in the controls it was $11.78( \pm 2.44) \mathrm{fL}$, which was statistically significant. Our findings are comparable with the other studies. ${ }^{[3,4,5,11,13]}$

Significant positive correlation was found between fasting blood sugar and PDW. ${ }^{[9,10,13]}$ Kamilla et al observed a positive correlation between fasting blood sugar and PDW in diabetic patients, but Pearson correlation was not significant in controls. ${ }^{[13]}$ Agrawal A et al observed negative correlation with fasting blood sugar which was statistically significant. ${ }^{[16]}$ In the present study, PDW in controls showed a negative correlation with fasting blood sugar, which is statistically significant. (Rho $=-0.287$, $p=0.026, \mathrm{~N}=60$ ), while in cases, PDW shows a positive correlation with fasting blood sugar. However, it is not statistically significant. ( $\mathrm{Rho}=0.021, p=0.874, \mathrm{~N}=60$ ). A strong positive correlation was found between PDW and HbA1c levels. ${ }^{[3,8,9,10,13]}$

Significant increases were observed with various grades of HbA1c levels. ${ }^{[9]}$ PDW was significantly higher in the diabetics with $\mathrm{HbA} 1 \mathrm{c}>7 \%$, when compared to diabetics with $\mathrm{HbA} 1 \mathrm{C} \leq 7 \%{ }^{[12]}$ In patients with both microvascular and macrovascular complications, there was a statistically significant correlation between PDW and HbAlc levels. ${ }^{[13]}$ Using Odds ratio, we calculated the association of $\mathrm{HbA} 1 \mathrm{c}$ and PDW. For PDW the Odds ratio calculated was 84.00 , with $95 \%$ CI ranging between 8.16 to 863.70 . The significance level was $\mathrm{P}=0.0002$. This indicates strong association between PDW and $\mathrm{H}$ bA1c levels. This is in agreement with the various studies. ${ }^{[3,8,9,10,13]}$

Jaman S et al calculated the sensitivity of MPV to be $85.19 \%$ and specificity to be $30.30 \%$ with a positive predictive value of $58.23 \%$ with a cut off of $9.55 \mathrm{fL} .^{[8]}$ while Damira K et al observed that at the best cut off value 9.55fL, MPV showed a sensitivity of $82 \%$ and a specificity of $54.5 \%$ with a positive predictive value of $71.8 \% .^{[17]}$ In our study, at a cut off of $9.5 \mathrm{fL}$ MPV showed a sensitivity of $78.33 \%$, specificity of $70 \%$ and a PPV of $72.30 \%$. Thus, our results are comparable with those of Jaman $\mathrm{S}$ et al and Damira K et al. At a cut off of $14.55 \mathrm{fL}$, PDW showed a sensitivity of $45 \%$, specificity of $67.86 \%$ and a positive predictive value of $75 \%$ in a study carried out by Jaman $S$ et al. ${ }^{[8]}$ In the present study, PDW showed a sensitivity of $81.66 \%$, specificity of $91.66 \%$ and a PPV of $90.74 \%$ at a cut-off of $15 \mathrm{fL}$.

Various studies state that MPV and PDW can be used as simple and cost effective prognosticators of deterioration of glucoregulation. They cannot be considered as a substitute for $\mathrm{HbAlc}$, but in situations of financial constraints, they can be used as biomarkers for early detection of impending complication. ${ }^{[3,5,7,9,10,11,12,13]}$ The results of our study prove that PDW is a more specific indicator to monitor patients with diabetes mellitus, This is in agreement with studies carried out by Vagdatli et al, Rechenski et al, Jindal et al and Yilmaz et al. ${ }^{[18,19,20,21]}$

\section{Conclusion}

Changes in Platelet parameters are seen in patients with Diabetes Mellitus. Most of the automated haematology cell counters give the Platelet indices consisting of MPV (mean platelet volume), PDW (platelet distribution width), Pct (Plateletcrit) and P-LCR (Platelet large cell ratio) along with Platelet count. However, only Platelet count is widely used as an investigative tool. In the present study, we evaluated two platelet indices MPV and PDW. At a cut off of $9.5 \mathrm{fL}$, MPV had a sensitivity of $78.33 \%$, specificity of $70 \%$ and a positive predictive value of $72.30 \%$. At a cut off of $15 \mathrm{fL}$, PDW showed a sensitivity of $81.66 \%$, specificity of $91.66 \%$ and a positive predictive value of $90.74 \%$. This proves that PDW is a independent risk factor as compared to MPV. The present study shows strong association between PDW and HbA1c and so PDW can be used as an indicator of impending vascular events

\section{Acknowledgements}

This study was carried out as a project under ICMR- STS and is approved. It was a learning experience, particularly application of statistical analysis to evaluate the efficacy of the platelet parameters under study. A sincere thanks to Dr. S.S.Raje and Mr. Anirudha N Joshi for helping us with the statistical analysis of the data. We are also grateful to the Management, our Principal Dr. Rajendra Prasad Gupta, Head of the Department of Pathology Dr. Sneha Joshi and the other faculty members for their constant encouragement, support and guidance. 


\section{Reference}

1. Bharathi K. Study of hematological profile and its significance in type 2 diabetes mellitus patients. Journal of Diagnostic Pathology and Oncology, July-September, 2016; 1(1):14-17

2. Anirban M.K, Abbas and Aster R and Cotran Pathologic Basis of Disease: South Asia Edition. Vol 2. Chapter 24.The Endocrine System. Elsevier;2015:1105-20

3. Jabeen F, Rizvi HA, Aziz F, Wasti AZ. Hyperglycemic induced variations in Hematological Indices in Type 2 Diabetics. IJAR. 2013; 1(8): 322-334.

4. Biadgo B, Melku M, Mekonnen Abebe S, Abebe M. Hematological indices and their correlation with fasting blood glucose level and anthropometric measurements in type 2 diabetes mellitus patients in Gondar, Northwest Ethiopia. Diabetes, Metabolic Syndrome and Obesity: Targets and Therapy 2016;9:91-99

5. Bhanukumar M, Ramaswamy PKH, Peddi NK, Menon VB. Mean Platelet Volume and Platelet Distribution Width as Markers of Vascular Thrombosis in Type 2 Diabetes Mellitus.J Postgrad Med Edu Res 2016;50(3):127-131

6. Nahla A. M. Hamed. Alterations in Hematological Parameters: Could it be a Marker in Diabetes Mellitus? BAOJ Diabet.2016; 2(1): 009.

7. Buch A, Kaur S, Nair R, Jain A. Platelet volume indices as predictive biomarkers for diabetic complications in Type 2 diabetic patients. J Lab Physicians 2017; 9 (2):84-88

8. 8. Jaman S, Swagat R, Alam S,Islam R,Husna A,Sayeed M. Association of Mean Platelet Volume and Platelet Distribution Width with HbA1c. J Endocrinol Diab.2017; 4(4): 1-6

9. Rajagopal L , Arunachalam S, Abdullah SM, Ganesan V, Kathamuthu K, Ramraj B.JCDR.2018;12(2):ECO1-ECO5

10. Sharma M, Narang S, Nema SK. Study of altered platelet morphology with changes in glycemic status. Int J Res Med Sci.2016; 4(3): 757-761.

11. Ansari JN, Buch AC, Supreet Kaur, Nikam SR, Nair R, Rajpal M. Co-relation of Platelet Volume Indices with Lipid Profile in Diabetic and Non Diabetic Patients: A Case Control Study. Ejpmr. 2017; 4(6):565-569.

12. Anandhalakshmi S, Kalaivani A, Shivasekar G, Saravanan A. Evaluation of Mean platelet volume and other platelet parameters in subjects with Type 2 Diabetes Mellitus. Natl J Physiol Pharm Pharmacol. 2017; 7(1): 51-54.

13. lhadas KR, Santos SN, Freitas MM, Viana SM,Ribeiro LC,Costa MB. Are Platelet indices useful in the evaluation of Type 2 diabetic patients? J Bras Patol Med Lab.2016; 52(2):96-102.

14. Shah B, Sha D, Xie D,Mohler ER, Berger JS. The relationship between Diabetes, Metabolic Syndrome, and Platelet activity as measured by Mean Platelet Volume. Diabetes Care. 2012; 35: 1074-1078.

15. Akinsegun A, Olusola DA, John- Olabode S et al. Mean Platelet Volume and platelet counts in type 2 Diabetes Mellitus on treatment and non-diabetic mellitus controls in Lagos, Nigeria. Pan African Medical Journal.2014;18:42.

16. Agrawal A, Kumar S, Bhagwati J. Correlation of Platelet Indices with Clinical Profile in Elderly Patients: Study in Rural Teaching Hospital. Ann Med Health Sci Res. 2018; 8:163-169.

17. Damira Kadic, Hasic S, Spahic E. Mean Platelet Volume predicts the glycemic control deterioration in diabetes mellitus type 2 patients. Medicinski Glasnik. 2016;13(1):17.

18. Vagdatli E, Gounari E, Lazaridou E, Katsibourlia E, Tsikopoulou F. Labrianou I.Platelet distribution width: a simple, practical and specific marker of coagulation. Hippokratia. 2010; 14(1):28-32

19. Rechcinski T, Jasinska A, Forys J et al. Prognostic value of platelet indices after acute myocardial infarction treated with primary percutaneous coronary intervention. Cardiol J.2013; 20 (5):491-8

20. Jindal S, Gupta S, Gupta R et al. Platelet indices in diabetes mellitus : indicators of diabetic microvascular complications. Haematology.2011; 16 (2):86-9

21. Yilmaz T, Yilmaz A. Relationship between Altered Platelet Morphological Parameters and Retinopathy in Patients with Type 2 Diabetes Mellitus. J Ophthalmol.2016;2016:9213623.

22. Aleksandra Korniluk, Olga Martyna Koper-Lenkiewicz et al. Mean Platelet Volume (MPV): New Perspectives for an Old Marker in the course and prognosis of inflammatory conditions.Mediators Inflamm.2019;2019:9213074.

23. Yuce S, Cure MC,Cure E,Kiztanir S, Yazici T. Evaluation of mean platelet volume before and after iron deficiency anaemia treatment. Int Med J Sifa Univ 2015;2:7-10.

24. S Periasamy, Adhin Antony Xavier, Gowtham R. Incidence of anaemia in type 2 diabetic mellitus and its prognostic index. Int J Med Res Rev 2016;4 (7):1239-1242.

25. Zsori KS, Mahalek JM,Mokarrami MA et al. Preanalytical factors affecting the Mean Platelet Volume: a Review. Global Journal of Hematology and Blood Transfusion.2016;3:19-22.

*Corresponding author:

Dr. Janice Jaison, Flat no 2, Jai Apartments, Plot no 121, Sect 26, Nigdi, Pradhikaran, Pune-411044 India

Phone: +917588330968

Email: drjanicej@gmail.com

Financial or other Competing Interests: None. 